Sharif University of Technology
Scientia Iranica
Transactions E: Industrial Engineering
hCIENTIA

\title{
Transient solution of multiple vacation queue with discouragement and feedback
}

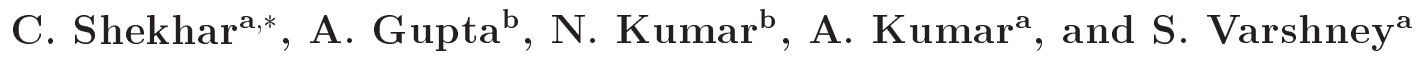 \\ a. Department of Mathematics, Birla Institute of Technology and Science, Pilani Campus, Pilani, Rajasthan, 333 031, India. \\ b. Department of Computer Science and Engineering, Uttarakhand Technical University, Dehra Dun, Uttarakhand, 248 007, India. \\ Received 13 February 2019; received in revised form 17 July 2020; accepted 3 October 2020
}

\author{
KEYWORDS \\ Multiple vacations; \\ Balking; \\ Reneging; \\ Feedback; \\ Confluent \\ hypergeometric \\ function; \\ Generating function; \\ Modified Bessel \\ function.
}

\begin{abstract}
Congestion problems with processor vacations are characterized by high intricacy and explicit transient solutions to these problems are exceptionally difficult to apply. These solutions are essential to studying the dynamic behavior of computing systems over a finite period and they are predominantly utilized within a state-of-theart design architecture for a real-time I/O system. Motivated by the above finding, this study adopts mathematical concepts, namely continued fractions and generating function, to derive explicit expressions for transient-state probabilities. Transient-state probabilities of the processing delay problem with a single processor, which adopts the multiple-vacation policy to save power consumption and thermal trip error with discouragement and feedback, are obtained in terms of modified Bessel functions using the properties of the confluent hypergeometric function. Given the inaccessibility of the processor, discouraging behaviors like balking and reneging on job requests are likely to appear. Routing back for the service feedback for the processed job request is also critical to maintaining Quality of Service (QoS). Considering the performance of the I/O system, the expected value of the state of the computing system using stationary queue-size distribution is derived.
\end{abstract}

(C) 2022 Sharif University of Technology. All rights reserved.

\section{Introduction}

Queues or waiting lines tend to exist at critical points in the job request/processor journey in I/O and computing systems. They may appear in service systems, computer and communication systems, manufacturing and production systems, check-in and check-out systems, etc. Irrespective of where queue occurs, they may perpetually find an opportunity to emphatically or adversely impact the experience of job requests and the proficiency of the processing

*. Corresponding author. Tel.: +91-9414492349

E-mail addresses: chandrashekhar@pilani.bits-pilani.ac.in (C. Shekhar); toamitji@gmail.com (A. Gupta);

neerajpundir@yahoo.com (N.Kumar); amitk251@gmail.com

(A. Kumar); skvarshney91@gmail.com (S. Varshney)

doi: 10.24200 /sci. 2020.52933 .2955 system. Not only the benefits of the well-managed queue are many, but also the negative effects of poor management on the improvement of the computing system are significant. A well-managed queue can reduce the impatient behavior (timeout) associated with job requests, improve the overall perception of the computing system, increase the conversion between job requests and processors, optimize service allocation, and encourage positive word of mouth. In contrast, a poorly managed queue reduces satisfaction of correctly processed job requests, diminishes job request loyalty, decreases repeat requests, and attenuates efficiency and productivity.

Availability of processing facilities is one of the critical issues of queue management in the I/O system. The scheduling of vacation of the processing facility is an essential feature of interest in a well-managed computing system to make a balance among idle time, 
extra cost, unavailability, power consumption, thermal trip error, etc. When the processing facility is off, the processor does not process the job requests and enters the sleeping mode or may be busy performing some secondary job requests. In general, the vacation schedule commences whenever there is no job request in the computing system for processing and it takes repeated vacations until there is at least one job request in the system to seek the processing facility at the end of the random duration of the vacation.

In case of the imbalance between the job request's waiting and processing facility, longer waiting, job request delay, blocking, rise in discouragements, and job request loss are experienced. Whenever the balance between job requests and processing facility is exposed to disruption, common reactions of job requests include the following:

(a) Impatient behaviors (timeout) like balking: A job request may decide that the queue is too long, thus leaving the processing facility;

(b) Reneging: A job request may join the processing facility, but become impatient and leave the facility without taking the intended process.

A well-managed computing system deals with such issues strategically to prevent job request loss and delay. These reluctant behaviors associated with job requests can be reduced or moderated through proper strategies like publishing the estimated waiting times, considering virtual computing, monitoring the queues in real time, etc.

The feedback loop for job requests about the satisfaction and dissatisfaction experienced in processing is critical. This information is an important source for the processing facility provider to improve and address the needs and demands of those applicants with job requests. Through feedback collection or inspection, a job request that has already received a unit of processing routes back to the waiting line and under some decision rules receives another unit of processing. The feedback or inspection procedures provide a computing framework for requests, jobs, or packets that must be reworked. A broad class of such computing systems appears in computer modeling with round-robin models and foreground-background models. In this paper, such a computing system is referred to as a queue with feedback.

The design of a well-managed processing system is perfected after much micro or macro analysis of the experiences gained during trials of the developing phases. The transient analysis is a prominent tool to develop a stable computing system. A designing framework is established from the beginning state to a steady state. This framework involves time-dependent tracking of the pros and cons of the system to finally determine proper input parameters and conditions to ensure better system output. Transient analysis is seldom available in the literature, even for the simple computing model, despite its usefulness and necessity in designing a better service system. This gap has motivated us to suggest a simple procedure to evaluate the transient solution to the issue of complex computing systems. For this purpose, this study establishes time-dependent queue-size distribution in terms of continued fraction using the properties of the confluent hypergeometric function.

The purpose of this study is threefold. The first purpose is to study the job applicant's discouraging behavior and feedback with respect to the computing system as well as the processor's multiple vacations. The second purpose is to identify the conditions for the existence and to derive the explicit expression of transient queue-size distribution in terms of continued fraction and modified Bessel function using properties of the confluent hypergeometric function. This objective is the kernel of this research. The third purpose is to compute the queue indices in terms of transient probabilities to give system performance at a glance for proper management and to validate the proposed procedure for some known results for existing models as a particular case.

The remainder of the paper is structured as follows. In Section 2, the literature review and survey are conducted to identify the research gap. In Section 3, the governing forward differential-difference equations are given along with prominent assumptions and notations for the proposed computing model. The properties of the confluent hypergeometric function are highlighted in Section 4. In Section 5, the transient queue-size distribution of the proposed model is generated. The expected value for the number of job requests in the system is derived using queue-size distribution in Section 6. In Section 7, a number of particular cases are presented to validate our findings. In Section 8 , the concluding remarks are summarized and the future directions and limitation are highlighted.

\section{Literature review}

Today, we live in the world of Internet of Things (IoT) technology or a fast-paced society. However, one remaining issue is 'waiting', which is a recurrent phenomenon at the multiplex, mall, bank or even communication, computer computing, or in different ways of travel. Well-managed computing systems can play a major role in the processing environment. Most packets, jobs, or requests are familiar with the pros and cons of each computing system. Different state-ofthe-art designs of computing systems can greatly affect the experience of data, requests, jobs, or consumers and, also, cause the problem of queue or waiting like 
reneging and balking. Many research pieces have contributed to the development of the design of a better computing system with different behavior types of job applicants and processor-oriented mechanism since the commencement of computing theory.

Specifically, many researchers have paid considerable attention to vacation queue and impatient behavior associated with job requests. An exhaustive survey of computing models with vacations was provided by Doshi [1], who presented an overview of some general decomposition results and the associated methodology. Over the last decade, many relevant new results have been obtained for the single and multiple processor queues with vacations (c.f., [2-9]). Thomasian [10] obtained the mean delay cycle for the $M / G / 1$ queueing system using the Vacationing Server Model (VSM) in which the delay cycle was initiated with arrival during a vacation and ended with an empty queue and then, a vacation restarted. Recently, Banik and Ghosh [11] analyzed the finite-buffer single processor computing system with vacation(s), assuming that the arrivals followed a Batch Markovian Arrival Process (BMAP) and went through processing according to a nonexhaustive gated-limited service discipline. Shekhar et al. [12] used a metaheuristic scheme to devise optimal strategies for emergency vacationing queueing model.

The impatient (timeout) behaviors of the customer(s), like balking and reneging, were first investigated by Haight $[13,14]$. After that, many researchers have used these revenue cut-throat job request behaviors in several real-time computing scenarios as mobile networking, computer communication, assembly system, machine repair problem, etc. (c.f., [1517]). Selvaraju and Goswami [18] derived a closed form of expressions for the transient-state probability distribution for the queueing model with the impatient behavior of the customer(s) and two different vacation policies (single and multiple). Lately, researchers have determined equilibrium conditions for different types of queueing circumstances as well as impatience of customers (c.f., [19-23]). Shekhar et al. [24] used a matrix-geometric technique to compute the queue-size distribution for queueing model with Bernoulli scheduled vacation and retention of the reneging customer.

In the past, many academicians or researchers expressed interests in presenting different and easyto-use approaches to determine queue-size distribution for different computing models with realistic design parameters using many different mathematical concepts. Time-dependent probabilities can be expressed in terms of Bessel functions (c.f., [25]). Varshney et al. [26] employed the diffusion approximation method to obtain transient probability distribution for the multi-server queueing model with balking. Al-Seedy et al. [27] obtained the closed-form queue size distribution using a generating function and some Bessel func- tion properties for the multi-server queueing model. Ammar et al. [28] discussed the single-server queue with a finite waiting space, discouraged arrivals and reneging, and obtained time-dependent probabilities in terms of the eigenvalues of a symmetric tridiagonal matrix. Ammar [29] provided an elegant explicit solution for the queue with two heterogeneous servers associated with impatient behavior. Using the generating function and Bessel function properties, Kumar and Sharma [30] explored the transient multiserver queue with balking and retention of reneging customers.

The feedback loop of the job requests is essential to the Quality of Service (QoS) in processing-based computing systems. Atencia and Moreno [31] analyzed a discrete-time $G e o^{[X]} / G_{H} / 1$ retrial queue in which each call after service either immediately returned to the orbit for another service with some probability or left the system forever with complementary probability. Several studies (c.f., [32-38]) existing in the literature have shown that the served customer or processed job provides feedback at a service completion instant due to unsatisfaction. Recently, Chang et al. [39] analyzed an unreliable server retrial queue with customers' feedback and impatience to investigate the economic viability of systems. With the help of the supplementary variable technique, Rajadurai et al. [40] obtained steady-state probabilities via generating function for the system size for a single-server feedback retrial queueing system with multiple working vacations and vacation interruption. Forghani and Fatemi Ghomi [41] explored the applicability of the processor-based queueing system in different sectors.

\section{Model description}

This study considers a single-processor computing system with First-Come First-Serve (FCFS) service discipline and realistic behavior of job requests and processor. The following assumptions and notations are considered to structure the proposed computing system and to develop the governing equations:

- From the infinite population of the prospective job requests, jobs arrive at the computing system following the Poisson process with mean arrival rate $\lambda$;

- If the processor is available and idle, the job request is immediately processed; otherwise, it adds the waiting queue to a space with infinite capacity;

- The job request processing time durations are independent and identically distributed (i.i.d.) exponential random variables with parameter $\mu$;

- For maintaining the state-of-the-art design and quality of the service, the processor seeks feedback from 
the served job requests. The processed job request leaves the computing system with the probability $\xi$ or routes back the queue to provide feedback with complementary probability $1-\xi$;

- On the completion of the services for all waiting job requests, the processor opts for a vacation to take a rest or to diminish idle time or rendering service cost or thermal break error or power consumption. The processor's vacation time is an exponential random variate with the meantime $1 / \theta$;

- At the epoch of the vacation end, if the computing system is empty, the processor recommences another vacation for random duration and repeatedly continues until the system has at least one job request waiting in the system;

- If the processor is not available for service due to vacation, some job requests may exhibit impatience (timeout) behavior. If the processor is on vacation, the job requests may balk with probability $\beta$ from the system without joining the computing system. The job requests may also renege without being served after waiting for some random periods when the processor is on vacation. The time-to-renege against the system follows an exponential distribution with meantime $1 / \eta$.

For modeling purposes, the state of the computing system is defined as follows:

$$
S(t) \equiv\left\{\begin{aligned}
& 0 ; \text { The processor is in vacation state } \\
& \text { at any instant of time } t \\
& 1 ; \text { The processor is in busy state at } \\
& \text { any instant of time } t
\end{aligned}\right.
$$

$$
\begin{aligned}
N(t) \equiv & \text { Number of the waiting job requests in } \\
& \text { the system at time } t .
\end{aligned}
$$

Hence, $\{(S(t), N(t)) ; t \geq 0\}$ is a Continuous Time Markov Chain (CTMC) on the state space $\Omega=$ $\{(0,0)\} \cup\{(s, n) ; s=0,1 \& n=1,2, \cdots\}$. Therefore, the governing transient-state probabilities are defined as follows:

$$
\begin{aligned}
& P_{0, n}(t)=\operatorname{Prob}\{S(t)=0, N(t)=n\} ; \quad n \geq 0, \\
& P_{1, n}(t)=\operatorname{Prob}\{S(t)=1, N(t)=n\} ; \quad n \geq 1 .
\end{aligned}
$$

Using the above-defined assumptions and notations, we develop the governing Chapman-Kolmogorov differential-difference equations of order and degree one for the studied model. On balancing the rates of inflow and outflow in each state, we have the following set of differential equations:

$$
\frac{d P_{0,0}(t)}{d t}=-\lambda P_{0,0}(t)+\eta P_{0,1}(t)+\xi \mu P_{1,1}(t),
$$

$$
\begin{aligned}
\frac{d P_{0,1}(t)}{d t}= & -(\lambda \bar{\beta}+\eta+\theta) P_{0,1}(t)+\lambda P_{0,0}(t) \\
& +2 \eta P_{0,2}(t) \\
\frac{d P_{0, n}(t)}{d t}= & -(\lambda \bar{\beta}+n \eta+\theta) P_{0, n}(t)+\lambda \bar{\beta} P_{0, n-1}(t) \\
& +(n+1) \eta P_{0, n+1}(t) ; \quad n \geq 2, \\
\frac{d P_{1,1}(t)}{d t}=- & (\xi \mu+\lambda) P_{1,1}(t)+\theta P_{0,1}(t)+\xi \mu P_{1,2}(t), \\
\frac{d P_{1, n}(t)}{d t}= & -(\xi \mu+\lambda) P_{1, n}(t)+\lambda P_{1, n-1}(t) \\
& +\xi \mu P_{1, n+1}(t)+\theta P_{0, n}(t) ; \quad n \geq 2 .
\end{aligned}
$$

The system of differential-difference equation (Eqs. (1)-(5)) with initial conditions $P_{0,0}(0)=1$ and $P_{s, n}(0)=0 ; s=0,1$ and $n \geq 1$, i.e., there is no job request in the computing system to be processed at time $t=0$, can be solved using Laplace transformation, properties of confluent hypergeometric function, modified Bessel function, and generating function to compute the transient-state probabilities.

\section{Confluent hypergeometric function}

This section presents the definition and properties of the special function and confluent hypergeometric function, used for deriving an explicit expression of transient queue-size distribution in terms of continued fraction for the studied computing problem with the processor's vacation and job request's feedback and discouragement. The confluent hypergeometric function is a solution to the confluent hypergeometric equation, which is a degenerate form of a hypergeometric differential equation in which two of the three regular singularities merge into irregular singularity. The confluent hypergeometric function, ${ }_{1} \mathrm{~F}_{1}(a ; c ; z)$, is defined as infinite sum:

$$
\begin{aligned}
{ }_{1} \mathrm{~F}_{1}(a ; c ; z) & =1+\frac{a}{c} \frac{z}{1 !}+\frac{a}{c} \frac{(a+1)}{(c+1)} \frac{z^{2}}{2 !}+\cdots \\
& =\sum_{k=0}^{\infty} \frac{(a)_{k}}{(c)_{k}} \frac{z^{k}}{k !} ; \quad c \neq 0,-1,-2, \cdots
\end{aligned}
$$

where $(\alpha)_{k}$ is the rising factorial function that can be represented as follows:

$$
(\alpha)_{k}=\frac{\Gamma(\alpha+1)}{\Gamma(\alpha-k+1)} ; \quad k=0,1,2, \cdots
$$

From the above definition, for $a=0$, we have:

$$
{ }_{1} \mathrm{~F}_{1}(0 ; c ; z)=1 \text {. }
$$

To generate the values of the function for different 
values of $a$ and $c$, the recurrent relation for the confluent hypergeometric function is as follows:

$$
\begin{gathered}
c(c-1){ }_{1} \mathrm{~F}_{1}(a-1 ; c-1 ; z)-a z{ }_{1} \mathrm{~F}_{1}(a+1 ; c+1 ; z) \\
=c(c-1-z){ }_{1} \mathrm{~F}_{1}(a ; c ; z) .
\end{gathered}
$$

It is established here that the quotient of two hypergeometric functions may be expressed as a continued fraction. It is useful for the algebra with a confluent hypergeometric function. The following identity is identified:

$$
\frac{{ }_{1} \mathrm{~F}_{1}(a+1 ; c+1 ; z)}{{ }_{1} \mathrm{~F}_{1}(a ; c ; z)}=\frac{c}{(c-z)+\frac{(a+1) z}{(c-z+1)+\frac{(a+2) z}{(c-z+2)+} \cdot}} .
$$

On simplification, it can be rewritten as follows:

$$
\begin{aligned}
& \frac{c{ }_{1} \mathrm{~F}_{1}(a ; c ; z)}{{ }_{1} \mathrm{~F}_{1}(a+1 ; c+1 ; z)}-(c-z) \\
&=\frac{(a+1) z}{(c-z+1)+\frac{(a+2) z}{(c-z+2)+\frac{(a+3) z}{(c-z+3)+}}} .
\end{aligned}
$$

For the confluent hypergeometric function, we have the following results:

$\sum_{k=0}^{\infty} \frac{(a)_{k}}{(c)_{k}} \frac{y^{k}}{k !}{ }_{1} \mathrm{~F}_{1}(a+k ; c+k ; x)={ }_{1} \mathrm{~F}_{1}(a ; c ; x+y)$.

Some values of $a$ and $c$ yield solutions that can be expressed in terms of other known functions. When $a$ is a non-positive integer, Kummer's confluent hypergeometric function, if defined, is a generalized Laguerre polynomial. Given that the confluent differential equation is a limit of the hypergeometric differential equation as the singular point at 1 is moved towards the singular point at $\infty$, the confluent hypergeometric function can be given as a limit of the hypergeometric function.

\section{Transient analysis}

This section derives an explicit expression for transient queue-size distribution for the computing system under consideration using the mathematical concepts of generating function, continued fractions, and some properties of confluent hypergeometric function. For this purpose, the following sequel is used.

\subsection{Laplace transform}

Laplace transform is an integral transform that converts a function of a real variable $t$ into a function of a complex variable $s$. It transforms differential equations into algebraic equations and convolution into multiplication. We define Laplace transformation of state probabilities and their derivatives as follows:

$$
\begin{array}{ll}
\widetilde{P}_{i, j}(s)=L\left(P_{i, j}(t)\right)=\int_{0}^{\infty} e^{-s t} P_{i, j}(t) d t ; & \forall i, j, \\
L\left(\frac{d P_{i, j}(t)}{d t}\right)=s \widetilde{P}_{i, j}(s)-P_{i, j}(0) ; & \forall i, j .
\end{array}
$$

Through the defined Laplace transform, the system of governing differential-difference equations is transformed as a system of linear equations as follows:

$$
\begin{aligned}
s \widetilde{P}_{0,0}(s)-1= & -\lambda \widetilde{P}_{0,0}(s)+\eta \widetilde{P}_{0,1}(s)+\xi \mu \widetilde{P}_{1,1}(s), \\
s \widetilde{P}_{0,1}(s)= & -(\lambda \bar{\beta}+\eta+\theta) \widetilde{P}_{0,1}(s)+\lambda \widetilde{P}_{0,0}(s) \\
& +2 \eta \widetilde{P}_{0,2}(s), \\
s \widetilde{P}_{0, n}(s)= & -(\lambda \bar{\beta}+n \eta+\theta) \widetilde{P}_{0, n}(s)+\lambda \bar{\beta} \widetilde{P}_{0, n-1}(s) \\
& +(n+1) \eta \widetilde{P}_{0, n+1}(s) ; \quad n \geq 2, \quad(1) \\
s \widetilde{P}_{1,1}(s)=- & (\xi \mu+\lambda) \widetilde{P}_{1,1}(s)+\theta \widetilde{P}_{0,1}(s)+\xi \mu \widetilde{P}_{1,2}(s), \\
s \widetilde{P}_{1, n}(s)= & -(\xi \mu+\lambda) \widetilde{P}_{1, n}(s)+\lambda \widetilde{P}_{1, n-1}(s) \\
& +\xi \mu \widetilde{P}_{1, n+1}(s)+\theta \widetilde{P}_{0, n}(s) ; \quad n \geq 2 . \quad(1)
\end{aligned}
$$

5.2. Evaluation of $P_{1, n}(t), n \geq 1$ in terms of $P_{0, n}(t)$

Define the generating function:

$$
P(z, t)=\sum_{n=1}^{\infty} P_{1, n}(t) z^{n} .
$$

Using some algebraic manipulation with Eqs. (5) and (6), the following partial differential equation is established:

$$
\begin{aligned}
\frac{\partial P(z, t)}{\partial t}= & \left\{-(\lambda+\xi \mu)+\lambda z+\frac{\xi \mu}{z}\right\} P(z, t) \\
& +\theta \sum_{n=1}^{\infty} P_{0, n}(t) z^{n}-\xi \mu P_{1,1}(t),
\end{aligned}
$$

which, upon integration, gives:

$$
\begin{aligned}
P(z, t)= & \theta \int_{0}^{t} \sum_{m=1}^{\infty} P_{0, m}(y) z^{m} e^{-(\lambda+\xi \mu)(t-u)} e^{\left(\lambda z+\frac{\xi \mu}{z}\right)(t-u)} d u \\
& -\xi \mu \int_{0}^{t} P_{1,1}(u) e^{-(\lambda+\xi \mu)(t-u)} \\
& e^{\left(\lambda z+\frac{\xi \mu}{z}\right)(t-u)} d u .
\end{aligned}
$$

For $\alpha=2 \sqrt{\lambda \xi \mu}$ and $\gamma=\sqrt{\frac{\lambda}{\xi \mu}}$, it is well known from the theory of Bessel function that: 


$$
\frac{\widetilde{P}_{0, n}(s)}{\widetilde{P}_{0, n-1}(s)}=\frac{\lambda \bar{\beta}}{(s+\lambda \bar{\beta}+\theta+n \eta)-\frac{(n+1) \eta \lambda \bar{\beta}}{(s+\lambda \bar{\beta}+\theta+(n+1) \eta)-\frac{(n+2) \eta \lambda \bar{\beta}}{(s+\lambda \bar{\beta}+\theta+(n+2) \eta)-}}}
$$

Box I

$$
e^{\left(\lambda z+\frac{\xi \mu}{z}\right) t}=\sum_{n=-\infty}^{\infty}(\gamma z)^{n} I_{n}(\alpha t)
$$

where $I_{n}($.$) is the modified Bessel function of the first$ kind. Upon comparing the coefficient of the power of $z$, i.e., $z^{n} ; n=1,2, \ldots$, on both sides of Eq. (17), we have:

$$
\begin{aligned}
P_{1, n}(t) & =\theta \int_{0}^{t} \sum_{m=1}^{\infty} P_{0, m}(y) \gamma^{n-m} I_{n-m}(\alpha(t-u)) \\
& e^{-(\lambda+\xi \mu)(t-u)} d u+\xi \mu \int_{0}^{t} P_{1,1}(y) \gamma^{n} I_{n}(\alpha(t-u)) \\
& e^{-(\lambda+\xi \mu)(t-u)} d u ; \quad n=1,2, \ldots
\end{aligned}
$$

The above equation also holds for $n=-1,-2, \cdots$ with the left-hand side replaced by zero, i.e., $P_{1, n}(t)=0$. Using $I_{-n}()=.I_{n}($.$) for n=1,2, \cdots$, we have the following:

$$
\begin{aligned}
& \theta \int_{0}^{t} \sum_{m=1}^{\infty} P_{0, m}(u) \gamma^{-n-m} I_{n+m}(\alpha(t-u)) e^{-(\lambda+\xi \mu)(t-u)} d u \\
& +\xi \mu \int_{0}^{t} P_{1,1}(u) \gamma^{n} I_{n}(\alpha(t-u)) e^{-(\lambda+\xi \mu)(t-u)} d u=0 \\
& \quad n=1,2, \ldots
\end{aligned}
$$

Hence, Eqs. (18) and (19) give $P_{1, n}(t)$ in terms of $P_{0, n}(t)$ :

$$
\begin{aligned}
P_{1, n}(t)= & \theta \int_{0}^{t} e^{-(\lambda+\xi \mu)(t-u)} \sum_{m=1}^{\infty} \gamma^{n-m} P_{0, m}(y) \\
& \times\left\{I_{n-m}(\alpha(t-u))-I_{n+m}(\alpha(t-u))\right\} d u
\end{aligned}
$$

$$
n=1,2, \ldots
$$

\subsection{Evaluation of $P_{0, n}(t), n \geq 2$ in terms of $P_{0,0}(t)$}

In this sub-section, the expression for $P_{0, n}(t)$ is obtained as continued fraction using the identities of confluent hypergeometric function. Eq. (12) is structured as follows:

$$
\frac{\widetilde{P}_{0, n}(s)}{\widetilde{P}_{0, n-1}(s)}=\frac{\lambda \bar{\beta}}{(s+\lambda+\theta+n \eta)-(n+1) \eta\left\{\frac{\widetilde{P}_{0, n+1}(s)}{\widetilde{P}_{0, n}(s)}\right\}},
$$

which can be further structured as a continued fraction as shown in Box I. Using the identity equation (Eq. (8)) of the confluent hypergeometric function discussed in Section 4, the above expression can be expressed in terms of confluent hypergeometric function:

$$
\begin{aligned}
& \frac{\widetilde{P}_{0, n}(s)}{\widetilde{P}_{0, n-1}(s)}=\frac{\lambda \bar{\beta}}{\eta} \\
& \quad \frac{{ }_{1} \mathrm{~F}_{1}\left((n+1) ;\left(\frac{s+\theta}{\eta}+n+1\right) ;\left(-\frac{\lambda \bar{\beta}}{\eta}\right)\right)}{\left(\frac{s+\theta}{\eta}+n\right){ }_{1} \mathrm{~F}_{1}\left(n ;\left(\frac{s+\theta}{\eta}+n\right) ;\left(-\frac{\lambda \bar{\beta}}{\eta}\right)\right)} .
\end{aligned}
$$

On iterative substitution and algebraic manipulation, the expression for $\widetilde{P}_{0, n}(s)$ in terms of $\widetilde{P}_{0,0}(s)$ is obtained as follows:

$$
\begin{aligned}
& \widetilde{P}_{0, n}(s)=\left(\frac{\lambda \bar{\beta}}{\eta}\right)^{n} \frac{1}{\prod_{i=1}^{n}\left(\frac{s+\theta}{\eta}+i\right)} \\
& \quad \frac{{ }_{1} \mathrm{~F}_{1}\left((n+1) ;\left(\frac{s+\theta}{\eta}+n+1\right) ;\left(-\frac{\lambda \bar{\beta}}{\eta}\right)\right)}{{ }_{1} \mathrm{~F}_{1}\left(n ;\left(\frac{s+\theta}{\eta}+n\right) ;\left(-\frac{\lambda \bar{\beta}}{\eta}\right)\right)} \widetilde{P}_{0,0}(s) ; \\
& n=2,3, \ldots \\
& \widetilde{P}_{0, n}(s)=\widetilde{\Psi}_{n}(s) \widetilde{P}_{0,0}(s) ; \quad n=2,3, \ldots
\end{aligned}
$$

On taking the inverse Laplace transform, we have:

$$
P_{0, n}(t)=\Psi_{n}(t) * P_{0,0}(t) ; \quad n=2,3, \ldots
$$

where $\Psi_{n}(t)$ is the inverse Laplace transform of $\widetilde{\Psi}_{n}(s)$, which will be evaluated in Subsection 5.6, and * represents the convolution between the two functions.

\subsection{Evaluation of $P_{0,0}(t)$}

From Eq. (10), we obtain:

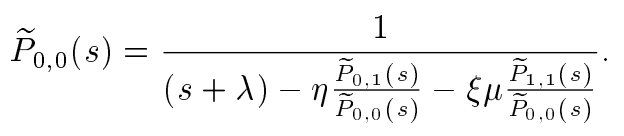

After putting $n=1$ into Eq. (19), we have the following expressions: 


$$
\begin{aligned}
P_{1,1}(t)= & \theta \int_{0}^{t} e^{-(\lambda+\xi \mu)(t-u)} \sum_{m=1}^{\infty} \gamma^{1-m} P_{0, m}(y) \\
& \times\left\{I_{1-m}(\alpha(t-u))-I_{1+m}(\alpha(t-u))\right\} d u
\end{aligned}
$$

and:

$$
P_{0,1}(t)=\Psi_{2}(t) * P_{0,0}(t),
$$

respectively. Hence, Eqs. (25) and (26) give:

$$
\begin{aligned}
\widetilde{P}_{0,0}(s)= & \sum_{k=0}^{\infty} \sum_{r=0}^{k}(-1)^{k} \theta^{k}\left(\begin{array}{l}
k \\
r
\end{array}\right)\left(\frac{\eta}{\theta}\right)^{k} \frac{\widetilde{\Psi}_{2}^{r}(s)}{(s+\lambda)^{k+1}} \\
& \left\{\sum_{m=1}^{\infty}\left(\frac{\chi-\sqrt{\chi^{2}-\alpha^{2}}}{\alpha \gamma}\right)^{m} \widetilde{\Psi}_{m}(s)\right\}^{k-r}
\end{aligned}
$$

where $\chi=(s+\lambda \beta+\xi \mu)$ which, upon inverse Laplace transform, yields:

$$
\begin{gathered}
P_{0,0}(t)=\xi \mu \sum_{k=0}^{\infty} \sum_{r=0}^{k}(-1)^{k} \theta^{k}\left(\begin{array}{l}
k \\
r
\end{array}\right)\left(\frac{\eta}{\theta}\right)^{k} e^{-\lambda t} \frac{t^{k}}{k !} \\
* \Psi_{2}^{r}(t) *\left\{\sum _ { m = 1 } ^ { \infty } \gamma ^ { 1 - m } \left(I_{m}(\alpha(t-y))\right.\right. \\
\left.\left.-I_{m+2}(\alpha(t-y))\right) \times e^{-(\lambda+\xi \mu) t} * \Psi_{m}(t)\right\}^{*(k-r)},
\end{gathered}
$$

where $*$ denotes the convolution, while $*(k-r)$ stands for the $(k-r)$-times convolution.

\subsection{Evaluation of $P_{0,1}(t)$}

Similarly, we obtain the algebraic expression of $P_{0,1}(t)$. From Eq. (11), we have:

$$
\begin{aligned}
(s+\lambda \bar{\beta} & +\eta+\theta) \widetilde{P}_{0,1}(s)=\lambda \widetilde{P}_{0,0}(s)+2 \eta \widetilde{P}_{0,2}(s) \\
\widetilde{P}_{0,1}(s)= & \frac{\lambda}{(s+\lambda \bar{\beta}+\eta+\theta)} \widetilde{P}_{0,0}(s) \\
& +\frac{2 \eta}{(s+\lambda \bar{\beta}+\eta+\theta)} \widetilde{P}_{0,2}(s)
\end{aligned}
$$

which, upon inverse Laplace transform, yields:

$$
\begin{aligned}
P_{0,1}(t)= & \lambda e^{-(\lambda \bar{\beta}+\theta+\eta) t} * P_{0,0}(t) \\
& +2 \eta e^{-(\lambda \bar{\beta}+\theta+\eta) t} * P_{0,2}(t) .
\end{aligned}
$$

\subsection{Evaluation of $\Psi_{n}(t) ; n \geq 2$}

Finally, for obtaining all transient-state probabilities, we should derive the expression for $\Psi_{n}(t)$. From Eq. (22), we have:

$$
\begin{aligned}
& \widetilde{\Psi}_{n}(s)=\left(\frac{\lambda \bar{\beta}}{\eta}\right)^{n} \frac{1}{\prod_{i=1}^{n}\left(\frac{s+\theta}{\eta}+i\right)} \\
& \frac{{ }_{1} \mathrm{~F}_{1}\left((n+1) ;\left(\frac{s+\theta}{\eta}+n+1\right) ;\left(-\frac{\lambda \bar{\beta}}{\eta}\right)\right)}{{ }_{1} \mathrm{~F}_{1}\left(n ;\left(\frac{s+\theta}{\eta}+n\right) ;\left(-\frac{\lambda \bar{\beta}}{\eta}\right)\right)} ; \quad n \geq 2 . \\
&(31)
\end{aligned}
$$

By using the definition of Eq. (7) for confluent hypergeometric function, we have:

$$
\begin{gathered}
\frac{{ }_{1} \mathrm{~F}_{1}\left((n+1) ;\left(\frac{s+\theta}{\eta}+n+1\right) ;\left(-\frac{\lambda \bar{\beta}}{\eta}\right)\right)}{\prod_{i=1}^{n}\left(\frac{s+\theta}{\eta}+i\right)} \\
=\eta^{n} \sum_{k=0}^{\infty} \frac{\left(\begin{array}{c}
n+k \\
k
\end{array}\right)(-\lambda \bar{\beta})^{k}}{\prod_{i=1}^{n+k}(s+\theta+i \eta)} ; \quad n \geq 2,
\end{gathered}
$$

which can be written into partial fractions as follows:

$$
\begin{gathered}
\frac{{ }_{1} \mathrm{~F}_{1}\left((n+1) ;\left(\frac{s+\theta}{\eta}+n+1\right) ;\left(-\frac{\lambda \bar{\beta}}{\eta}\right)\right)}{\prod_{i=1}^{n}\left(\frac{s+\theta}{\eta}+i\right)} \\
=\eta^{n} \sum_{k=0}^{\infty}\left(\begin{array}{c}
n+k \\
k
\end{array}\right)\left(\frac{-\lambda \bar{\beta}}{\eta}\right) \sum_{i=1}^{k} \\
\frac{(-1)^{i-1}}{(i-1) !(n+k-i) !} \frac{1}{s+\theta+i \eta} ; \quad n \geq 2 .
\end{gathered}
$$

We also have:

$$
\begin{aligned}
& { }_{1} \mathrm{~F}_{1}\left(1 ; \frac{s+\theta}{\eta}+1 ; \frac{-\lambda \bar{\beta}}{\eta}\right)=\sum_{k=0}^{\infty} \frac{(-\lambda \bar{\beta})^{k}}{\prod_{i=1}^{k}(s+\theta+i \eta)} \\
& =\sum_{k=0}^{\infty}(-\lambda \bar{\beta})^{k} \widetilde{a}_{k}(s)
\end{aligned}
$$

where:

$$
\begin{aligned}
\tilde{a}_{0}(s) & =1, \text { and } \\
\widetilde{a}_{k}(s) & =\frac{1}{\prod_{i=1}^{k}(s+\theta+i \eta)} \\
& =\frac{1}{\eta^{k-1}} \sum_{r=1}^{k} \frac{(-1)^{r-1}}{(r-1) !(k-r) !} \frac{1}{s+\theta+r \eta} \\
k & =1,2, \ldots
\end{aligned}
$$

Using the identity equation (Eq. (6)) from Section 4 for confluent hypergeometric function, we have: 


$$
\begin{gathered}
\left\{{ }_{1} \mathrm{~F}_{1}\left(n+1 ; \frac{s+\theta}{\eta}+n+1 ; \frac{-\lambda \bar{\beta}}{\eta}\right)\right\}^{-1} \\
=\sum_{k=0}^{\infty} \widetilde{b}_{k}(s)(\lambda \bar{\beta})^{k} ; \quad n \geq 2,
\end{gathered}
$$

where:

$$
\widetilde{b}_{k}(s)=1,
$$

and:

$\widetilde{b}_{k}(s)$

$$
=\left|\begin{array}{cccccc}
\widetilde{a}_{1}(s) & 1 & & \cdots & & \\
\widetilde{a}_{2}(s) & \widetilde{a}_{1}(s) & 1 & \cdots & & \\
\widetilde{a}_{3}(s) & \widetilde{a}_{2}(s) & \widetilde{a}_{1}(s) & \cdots & & \\
\vdots & \vdots & \vdots & \cdots & & \\
\widetilde{a}_{k-1}(s) & \widetilde{a}_{k-2}(s) & \widetilde{a}_{k-3}(s) & \cdots & \widetilde{a}_{1}(s) & 1 \\
\widetilde{a}_{k}(s) & \widetilde{a}_{k-1}(s) & \widetilde{a}_{k-2}(s) & \cdots & \widetilde{a}_{2}(s) & \widetilde{a}_{1}(s)
\end{array}\right| ;
$$$$
k=1,2, \ldots
$$

$\widetilde{b}_{k}(s)=\sum_{i=1}^{k}(-1)^{i-1} \widetilde{a}_{i}(s) \widetilde{a}_{k-1}(s) ; \quad k=1,2, \ldots$

Hence, substituting the results of Eqs. (33) and (36), into Eq. (31) gives:

$$
\begin{aligned}
& \widetilde{\Psi}_{n}(s)=(\lambda \bar{\beta})^{j}\left(\begin{array}{c}
n+j \\
j
\end{array}\right) \widetilde{a}_{n+j}(s) \sum_{k=1}^{\infty}(\lambda \bar{\beta})^{k} \widetilde{b}_{k}(s) ; \\
& n \geq 2,
\end{aligned}
$$

which, upon inverse Laplace transform, gives a required expression as follows:

$$
\begin{aligned}
& \Psi_{n}(t)=(\lambda \bar{\beta})^{j}\left(\begin{array}{c}
n+j \\
j
\end{array}\right) a_{n+j}(t) * \sum_{k=1}^{\infty}(\lambda \bar{\beta})^{k} b_{k}(t) ; \\
& n \geq 2,
\end{aligned}
$$

where:

$$
\begin{aligned}
a_{k}(t) & =\frac{1}{\eta^{k-1}} \sum_{r=1}^{k} \frac{(-1)^{r-1}}{(r-1) !(k-r) !} e^{-(\theta+r \eta) t} \\
k & =1,2, \ldots \\
b_{k}(t) & =\sum_{i=1}^{k}(-1)^{i-1} a_{i}(t) * b_{k-1}(t) \\
k & =2,3, \ldots \quad \text { and } \quad b_{1}(t)=a_{1}(t) .
\end{aligned}
$$

\section{Performance measure}

Systematic observation of the state of the computing system is vital to enhance the performance and improve decision-making. In the case of the computing system, performance index is primarily delivered through the expected value of the number of the job requests. Let:

$$
N(t) \equiv \begin{aligned}
& \text { Number of job requests in the computing } \\
& \text { system at time } t \text {. }
\end{aligned}
$$

Hence, the expected number of job requests in the computing system at time $t$ is:

$$
E(N(t))=\sum_{n=1}^{\infty} n\left(P_{0, n}(t)+P_{1, n}(t)\right) .
$$

Initially, at $t=0$, we assume that there is no job request to be processed in the computing system. Hence:

$$
E(N(0))=\sum_{n=1}^{\infty} n\left(P_{0, n}(0)+P_{1, n}(0)\right)=0 .
$$

Upon differentiating Eq. (42) with respect to $t$, we have:

$$
\frac{d E(N(t))}{d t}=\sum_{n=1}^{\infty} n\left(\frac{P_{0, n}(t)}{d t}+\frac{P_{1, n}(t)}{d t}\right) .
$$

Substituting the value from Eq. (1) into Eq. (5) and using mathematical manipulation, we get the following differential equation from Eq. (43):

$$
\begin{aligned}
& \frac{d E(N(t))}{d t}=\lambda P_{0,0}(t)+\lambda \bar{\beta} \sum_{n=1}^{\infty} P_{0, n}(t) \\
& -\eta \sum_{n=1}^{\infty} n P_{0, n}(t)+(\lambda-\xi \mu) \sum_{n=1}^{\infty} P_{1, n}(t),
\end{aligned}
$$

which, upon integration, gives:

$$
\begin{gathered}
E(N(t))=\lambda \int_{0}^{t} P_{0,0}(y) d y+\lambda \bar{\beta} \sum_{n=1}^{\infty} \int_{0}^{t} P_{0, n}(y) d y \\
-\eta \sum_{n=1}^{\infty} \int_{0}^{t} n P_{0, n}(y) d y+(\lambda-\xi \mu) \sum_{n=1}^{\infty} \int_{0}^{t} P_{1, n}(y) d y,
\end{gathered}
$$

where the explicit expressions for $P_{0, n}(t)$ and $P_{1, n}(t)$ have already been derived in Eqs. (20) and (23).

\section{Special cases}

For the justification and docility of the studied computing system, we have relaxed one or more assumptions and found that our model is similar to the models available in the existing literature. The viability of the analytical proof is not possible due to its complexity, even if more assumptions are relaxed.

The following are some special cases that we can be derived from the developed model after eliminating one or more assumptions:

Case 1: For $\xi=1$, i.e., the job request leaves the system after being processed without feedback loop. 
The proposed model and results coincide with the results of the model proposed in [42]. The model is reduced into a single-server computing model with impatient behavior and multiple vacations;

Case 2: By limiting the vacation parameter as $\theta \rightarrow 0$ and setting the feedback parameter as $\xi=1$, the proposed model resembles the computing model with balking and reneging proposed by the authors in [27]. They also computed the transient solution for queuesize distribution in a similar manner;

Case 3: If we set the balking probability $\beta=0$ and reneging parameter $\eta=0$, our model exhibits the result, which was obtained by Kalidass and Ramanath [43]. They studied $M / M / 1$ computing model with multiple vacations for server.

\section{Conclusion}

This study attempted to find a time-dependent solution for a single-processor Poisson queue in a computing system with job request feedback and discouragement (timeout) behaviors, namely balking and reneging and processor's multiple vacations. A closed-form explicit expression was derived for the queue-size distribution analytically by employing continued fractions, Laplace transforms, generating functions, and using the properties of confluent hypergeometric function. Then, a mean for the state of the computing system was established processing facility providers. The present study provided a basic idea so that queueing theorists would determine the transient solution of queueing problems and service providers use the design parameters for better services.

This research can be extended in the future with more realistic phenomena related to the waiting line problems like single and multiple vacations, Bernoulli vacation, working vacation, vacation interruption, imperfect service, multi-processor, processor breakdown, catastrophe, etc. Due to the computational complexity involved, many variants of the queue may not be applicable for its extension like jockeying, finite population, etc. For such variants, we must choose numerical techniques for deriving the transient queuesize distribution.

\section{Acknowledgement}

The authors would like to thank the editorial board and anonymous referees for the valuable constructive comments and suggestions on the earlier version of this paper.

\section{References}

1. Doshi, B. "Queueing systems with vacations-a survey", Queueing Systems, 1(1), pp. 29-66 (1986).
2. Ke, J.C., Wang, K.H., and Liou, C.H. "A single vacation model $G / M / 1 / K$ with $N$ threshold policy", The Indian Journal of Statistics, 68(2), pp. 198-226 (2006).

3. Zhang, M. and Hou, Z. " $M / G / 1$ queue with single working vacation", Journal of Applied Mathematics and Computing, 39, pp. 221-234 (2012).

4. Wu, C.H. and Ke, J.C. "Multi threshold policy for a multi-server queue with synchronous single vacation", Mathematical and Computer Modelling, 57(5-6), pp. 1122-1130 (2013).

5. Jain, M., Shekhar, C., and Shukla, S. "Vacation queueing model for a machining system with two unreliable repairmen", International Journal of Operational Research, 20(4), pp. 469-491 (2014).

6. Wu, C.H. and Ke, J.C. "Multi server machine repair problems under a $(V, R)$ synchronous single vacation policy", Applied Mathematical Modelling, 38(7-8), pp. 2180-2189 (2014).

7. Shin, Y.W. "Algorithmic approach to Markovian multi-server retrial queues with vacations", Applied Mathematics and Computation, 250, pp. 287-297 (2015).

8. Yang, D.Y. and Wu, C.H. "Cost minimization analysis of a working vacation queue with $N$-policy and server breakdowns", Computers and Industrial Engineering, 82, pp. 151-158 (2015).

9. Takhedmit, B. and Abbas, K. "A parametric uncertainty analysis method for queues with vacations", Journal of Computational and Applied Mathematics, 312, pp. 143-155 (2017).

10. Thomasian, A. "Vacationing server model for $M / G / 1$ queues for rebuild computing in RAID5 and threshold scheduling for readers and writers", Information processing Letters, 135, pp. 41-46 (2018).

11. Banik, A.D. and Ghosh, S. "Efficient computational analysis of non-exhaustive service vacation queues: $B M A P / R / 1 / / N(\infty)$ under gated-limited discipline", Applied Mathematical Modelling, 68, pp. 540-562 (2019).

12. Shekhar, C., Varshney, S., and Kumar, A. "Optimal control of a service system with emergency vacation using bat algorithm", Journal of Computational and Applied Mathematics, 364, 112332 (2020). DOI: 10.1016/j.cam.2019.06.048

13. Haight, F.A. "Queuing with balking", Biometrika, 44(3-4), pp. 360-369 (1957).

14. Haight, F.A. "Queuing with reneging", Metrika, 2(1), pp. 186-197 (1959).

15. Ke, J.C. and Wang, K.H. "Cost analysis of the $M / M / R$ machine repair problem with balking, reneging, and server breakdowns", Journal of the Operational Research Society, 50(3), pp. 275-282 (1999).

16. Gans, N., Koole, G., and Mandelbaum, A. "Telephone call centers: Tutorial, review, and research prospects", Manufacturing and Service Operations Management, 5(2), pp. 79-141 (2003). 
17. Wang, K.H., Ke, J.B., and Ke, J.C. "Profit analysis of the $M / M / R$ machine repair problem with balking, reneging, and standby switching failures", Computers and Operations Research, 34(2), pp. 835-847 (2007).

18. Selvaraju, N. and Goswami, C. "Impatient customers in an $M / M / 1$ queue with single and multiple working vacations", Computers and Industrial Engineering, 65(2), pp. 207-215 (2013).

19. Guha, G., Goswami, V., and Banik, A.D. "Equilibrium balking strategies in renewal input batch arrival queues with multiple and single working vacation", Performance Evaluation, 94, pp. 1-24 (2015).

20. Bruneel, H. and Maertens, T. "A discrete-time queue with customers with geometric deadlines", Performance Evaluation, 85-86, pp. 52-70 (2015).

21. Guha, G., Goswami, V., and Banik, A.D. "Algorithmic computation of steady-state probabilities in an almost observable $G I / M / c$ queue with or without vacations under state dependent balking and reneging", $A p$ plied Mathematical Modelling, 40(5-6), pp. 4199-4219 (2015).

22. Yang, D.Y. and Wu, Y.Y. "Analysis of a finite-capacity system with working breakdowns and retention of impatient customers", Journal of Manufacturing Systems, 44(1), pp. 207-216 (2017).

23. Wang, Q. and Zhang, B. "Analysis of a busy period queuing system with balking, reneging and motivating”, Applied Mathematical Modelling, 64, pp. 480-488 (2018).

24. Shekhar, C., Varshney, S., and Kumar, A. "Matrixgeometric solution of multi-server queueing systems with Bernoulli scheduled modified vacation and retention of reneged customers: A meta-heuristic approach", Quality Technology \& Quantitative Management, 18(1), pp. 39-66 (2021).

25. Parthasarathy, P.R. and Sharafali, M. "Transient solution to the many-server Poisson queue: A simple approach", Journal of Applied Probability, 26(3), pp. 584-594 (1989).

26. Varshney, K., Jain, M., and Sharma, G.C. "A multiserver queueing model with balking and reneging via diffusion approximation", Journal of Physical and Natural Science, 10, pp. 10-15 (1989).

27. Al-Seedy, R.O., El-Sherbiny, A.A., El-Shehawy, S.A., et al. "Transient solution of the $M / M / c$ queue with balking and reneging", Computers and Mathematics with Applications, 57(8), pp. 1280-1285 (2009).

28. Ammar, S.I., El-Sherbiny, A.A., El-Shehawy, S.A., et al. "A matrix approach for the transient solution of an $M / M / 1 / N$ queue with discouraged arrivals and reneging", International Journal of Computer Mathematics, 89(4), pp. 482-491 (2012).
29. Ammar, S.I. "Transient analysis of a twoheterogeneous servers queue with impatient behavior", Journal of the Egyptian Mathematical Society, 22(1), pp. 90-95 (2014).

30. Kumar, R. and Sharma, S. "Transient analysis of an $M / M / c$ queuing system with balking and retention of reneged customers", Communications in StatisticsTheory and Methods, 47(6), pp. 1318-1327 (2018).

31. Atencia, I. and Moreno, P. "Discrete-time $G e o^{[X]} /$ $G_{H} / 1$ retrial queue with Bernoulli feedback", Computers and Mathematics with Applications, 47(8-9), pp. 1273-1294 (2004).

32. Mitrani, I. and Robert, P. "On the ASTA property in a feedback processor-sharing queue", Performance Evaluation, 58, pp. 81-85 (2004).

33. Kumar, B.K., Rukmani, R., and Thangaraj, V. "On multiserver feedback retrial queue with finite buffer", Applied Mathematical Modelling, 33(4), pp. 2062-2083 (2009).

34. Liu, Z. and Gao, S. "Discrete-time $G e o_{1}, G e o_{2}^{X} / G_{1}$, $G_{2} / 1$ retrial queue with two classes of customers and feedback", Mathematical and Computer Modelling, 53(5-6), pp. 1208-1220 (2011).

35. Admas, R.V. "Infinitesimal perturbation analysis of a multi-stage tandem of fluid queue with additive loss feedback", Systems and Control Letters, 66, pp. 22-27 (2014).

36. Upadhyaya, S. "Performance prediction of a discretetime batch arrival retrial queue with Bernoulli feedback", Applied Mathematics and Computation, 283, pp. 108-119 (2016).

37. Liu, Y. and Whitt, W. "Stabilizing performance in a service system with time-varying arrivals and customer feedback", European Journal of Operational Research, 256(2), pp. 473-486 (2017).

38. Shekhar, C., Kumar, A., and Varshney, S. "Modified Bessel series solution of the single server queueing model with feedback", International Journal of Computing Science and Mathematics, 10(3), pp. 313-326 (2017).

39. Chang, F.M., Liu, T.H., and Ke, J.C. "On an unreliable-server retrial queue with customer feedback and impatience", Applied Mathematical Modelling, 55, pp. 171-182 (2018).

40. Rajadurai, P., Saravanarajan, M.C., and Chandrasekaran, V.M. "A study on $M / G / 1$ feedback retrial queue with subject to server breakdown and repair under multiple working vacation policy", Alexandria Engineering Journal, 57(2), pp. 947-962 (2018).

41. Forghani, K. and Fatemi Ghomi, S.M.T. "A queueing theory-based approach to desinging cellular manufacturing systems", Scientia Iranica, 26(3), pp. 18651880 (2019).

42. Ammar, S.I. "Transient analysis of an $M / M / 1$ queue with impatient behavior and multiple vacations", $A p$ plied Mathematics and Computation, 260, pp. 97-105 (2015). 
43. Kalidass, K. and Ramanath, K. "Transient analysis of an $M / M / 1$ queue with multiple vacations", Pakistan Journal of Statistics and Operation Research, 10(1), pp. 121-130 (2014).

\section{Biographies}

Chandra Shekhar is a faculty member at BITS Pilani, India and is actively involved in research in the areas of queueing theory, computer and communication system, machine repair problem, reliability and maintainability, stochastic process, evolutionary computation, statistical analysis, and fuzzy set and logic. Besides attending, presenting scientific papers, and delivering invited talks, he has a number of research articles in these fields in journals of repute. $\mathrm{He}$ is also a member of the editorial board and a reviewer of many reputed journals, academic societies, and Doctoral Research Committee and Advisory board of many universities. Authorship of textbook on differential Equation goes to his credit.

Amit Gupta received his MTech degree from GGSIPU, Delhi, India in Computer Science and Engineering in 2008. He is currently pursuing a $\mathrm{PhD}$ in Computer Science and Engineering at the Uttarakhand Technical University, Dehradun, India. He presented many research papers in national and international conferences of repute. His research focuses on RAM analysis of software-hardware system and communication network.

Neeraj Kumar received his MTech degree in Computer Science and Engineering from GGSIPU, Delhi, India in 2008. $\mathrm{He}$ is a Research Scholar at the Department of Computer Science and Engineering, Uttarakhand Technical University, Dehradun, India. He has presented many research papers in national and international conferences of repute. His research interests include queueing theory, reliability, and maintainability of computer networks.

Amit Kumar earned his $\mathrm{PhD}$ degree from the Department of Mathematics, BITS Pilani, Pilani. He is TIFR qualified in 2011 and UGC-BSR fellow. He has presented many research papers in national and international conferences of repute. He has many published research articles in journals of repute. His research interests include queueing theory, reliability theory, and operations research.

Shreekant Varshney completed his $\mathrm{PhD}$ from the Department of Mathematics, BITS Pilani, Pilani. He is CSIR SRF/NET qualified. He has presented many research papers in national and international conferences of repute. Many research articles in journals of repute are accredited to him. His research interests is queueing theory, stochastic modeling, and operations research. 